\title{
Requirement of the transcription factor USF1 in bovine oocyte and early embryonic development
}

\author{
Tirtha K Datta ${ }^{1,2,4,{ }^{*}}$, Sandeep K Rajput ${ }^{1,2,{ }^{*}}$, Gabbine Wee ${ }^{1,2}$, KyungBon Lee ${ }^{1,2,5}$, Joseph K Folger ${ }^{1,2}$ \\ and George W Smith ${ }^{1,2,3}$ \\ ${ }^{1}$ Laboratory of Mammalian Reproductive Biology and Genomics, Michigan State University, East Lansing, Michigan \\ 48824, USA, Departments of ${ }^{2}$ Animal Science and ${ }^{3}$ Physiology, Michigan State University, East Lansing, Michigan \\ 48824, USA, ${ }^{4}$ Animal Genomics Laboratory, National Dairy Research Institute, Animal Biotechnology Centre, Karnal \\ 132001, Haryana, India and ${ }^{5}$ Department of Biology Education, College of Education, Chonnam National University, \\ Gwangju, Republic of Korea
}

Correspondence should be addressed to G W Smith; Email: smithge7@msu.edu

*(T K Datta and S K Rajput contributed equally to this work)

\begin{abstract}
Upstream stimulating factor 1 (USF1) is a basic helix-loop-helix transcription factor that specifically binds to E-box DNA motifs, known cis-elements of key oocyte expressed genes essential for oocyte and early embryonic development. However, the functional and regulatory role of USF1 in bovine oocyte and embryo development is not understood. In this study, we demonstrated that USF1 mRNA is maternal in origin and expressed in a stage specific manner during the course of oocyte maturation and preimplantation embryonic development. Immunocytochemical analysis showed detectable USF1 protein during oocyte maturation and early embryonic development with increased abundance at 8-16-cell stage of embryo development, suggesting a potential role in embryonic genome activation. Knockdown of USF1 in germinal vesicle stage oocytes did not affect meiotic maturation or cumulus expansion, but caused significant changes in mRNA abundance for genes associated with oocyte developmental competence. Furthermore, siRNA-mediated depletion of USF1 in presumptive zygote stage embryos demonstrated that USF1 is required for early embryonic development to the blastocyst stage. A similar (USF2) yet unique (TWIST2) expression pattern during oocyte and early embryonic development for related E-box binding transcription factors known to cooperatively bind USF1 implies a potential link to USF1 action. This study demonstrates that USF1 is a maternally derived transcription factor required for bovine early embryonic development, which also functions in regulation of JY1, GDF9, and FST genes associated with oocyte competence.
\end{abstract}

Reproduction (2015) 149 203-212

\section{Introduction}

In mammals, oocyte-specific genes play a key role in regulation of the reproductive process and are critical to both ovarian folliculogenesis and early embryogenesis (Rajput et al. 2013). The products of these genes which are expressed, stored, and secreted during oogenesis control the follicular microenvironment and promote oocyte competence for successful fertilization and the subsequent embryonic development (De Sousa et al. 1998). Several studies have revealed that aberrant expression of these maternal transcripts is directly associated with infertility due to either complete growth arrest or compromised development of oocyte and preimplantation embryos. For example, targeted deletion of Gdf9, expressed throughout most stages of folliculogenesis, causes a block in follicular development at the primary follicle stage and complete infertility in mice (Dong et al. 1996). Oocyte expressed GDF9 protein is also an important paracrine regulator of surrounding granulosa/cumulus cell functions, which in turn affects competency of the oocyte to develop into a blastocyst (BL) after fertilization (Mottershead et al. 2012, 2013, Peng et al. 2013). Similarly, the bovinespecific protein JY1 plays a functional role in regulation of follicular function and early embryogenesis (Lee et al. 2014a). Supplementation of bovine granulosa cell culture media with recombinant JY1 (rJY1) protein modulates follicle-stimulating hormone (FSH)-induced steroidogenesis. Depletion of $J Y 1$ transcripts in germinal vesicle (GV)-stage oocytes demonstrated adverse effects on nuclear maturation and expansion of surrounding cumulus cells, which retards early embryonic development after IVF. Similarly, siRNA-mediated knockdown of maternal $J Y 1$ transcripts in zygote-stage embryos demonstrated a functional requirement for JY1 after fertilization (Bettegowda et al. 2007). Supplementation 
of rJY1 protein during oocyte and embryo culture rescued the effect of $J Y 1$ knockdown on cumulus expansion and meiotic maturation and $\mathrm{BL}$ development respectively (Lee et al. 2014a). In addition to JY1, our laboratory has previously characterized the functional role of maternal (oocyte-derived) follistatin (FST) in promoting bovine early embryogenesis. Depletion of maternal FST in zygotes not only reduced the number of embryos developing to 8-16-cell stage and BL stage but also decreased the number of total and TE cells in BL (Lee et al. 2009).

Over the last decades, the functional role of numerous oocyte expressed/specific transcripts has been identified during folliculogenesis (Nobox, Figla, and c-Kit; Soyal et al. 2000, Rajkovic et al. 2004, Hutt et al. 2006), fertilization ( $Z p$ genes; Dean 1992), early cleavage (NALP, Zar1, and Npm2;; Tong et al. 2000, Burns et al. 2003, Wu et al. 2003, Peng et al. 2012), and embryonic genome activation (Brg1 and H1oo; Tanaka et al. 2001, Saeki et al. 2005, Bultman et al. 2006) using different gene knockdown strategies in mammals. The majority of oocyte-expressed genes involved in fertilization and embryonic development display highly conserved structural and functional properties among different mammalian species (Sylvestre et al. 2013). However, understanding of how these maternally expressed genes are transcriptionally regulated and hence fundamental mechanisms regulating oogenesis and early embryonic development remains poorly understood due to limited characterization of oocyte-expressed transcription factors in mammals. Comprehensive computational, mutational, and functional analysis of the promoters of several of these oocyte-specific genes including GDF9, JY1, FST, ZP genes, NALP genes, H1oo, and Npm2 revealed common cis-acting regulatory elements known as E-box, present within $200 \mathrm{bp}$ upstream of the transcription start site (TSS) and critically important for their transcriptional regulation during oogenesis (Liang et al. 1997, Yan et al. 2006, Bettegowda et al. 2007, Tsunemoto et al. 2008). Interestingly, this conserved and canonical E box (CANNTG) DNA element is a well-characterized binding site for $\mathrm{FIG} \alpha$, upstream stimulatory factor 1 (USF1), USF2 and TWIST2, and other transcription factors of the basic helix-loop-helix $(\mathrm{bHLH})$ and leucine zipper family. The functional role of oocyte-expressed FIG $\alpha$ has been well documented in transcriptional regulation of zona pellucida genes ( $Z P 1$, $Z P 2$, and ZP3) necessary for fertilization (Liang et al. 1997), and NALP family of genes (Joshi et al. 2007) essential for early embryonic development beyond the two-cell stage in mice (Tong et al. 2000). Moreover, E-box-dependent regulatory roles of USF1, USF2, and TWIST2 transcription factors have been clearly demonstrated in transcriptional regulation of genes associated with stress, immune responses, and the cell cycle and proliferation in different somatic and cancer tissues (Corre \& Galibert 2005, Xue \& Hemmings 2012). To our knowledge, the temporal expression and potential functional role of USF1, USF2, and TWIST2 in oocytes and early embryos have not been reported previously.

In this study, we investigated the temporal expression of USF1, USF2, and TWIST2 during oocyte and early embryogenesis in cattle. The functional role of USF1 in early embryonic development and transcriptional regulation of select oocyte-expressed genes containing an E-box motif in their promoter region was analyzed using siRNA-mediated gene knockdown in cumulusenclosed oocytes before initiation of meiotic maturation. Results support a potential regulatory role for abovementioned transcription factors in bovine oocyte and early embryonic development.

\section{Materials and methods}

All chemicals and reagents used were obtained from SigmaAldrich unless specified.

\section{In vitro oocyte and embryo culture}

In vitro maturation of oocytes, IVF, and embryo culture to the BL stage were conducted as described previously (Bettegowda et al. 2006). Briefly, cattle ovaries were obtained from a local slaughterhouse and cumulus-oocyte complexes (COCs) were aspirated from 2 to $7 \mathrm{~mm}$ antral follicles. GV-stage oocytes enclosed by a minimum of three compact layers of cumulus cells and containing homogenous cytoplasm were collected immediately after aspiration. COCs were cultured for $24 \mathrm{~h}$ in maturation medium (Medium-199, 10\% FBS; Gibco-BRL) containing $1 \mathrm{IU} / \mathrm{ml} \mathrm{FSH}, 5 \mathrm{IU} / \mathrm{ml}$ luteinizing hormone $(\mathrm{LH})$, and $1 \mu \mathrm{g} / \mathrm{ml}$ estradiol- $17 \beta$ to induce meiotic maturation. Matured oocytes were fertilized with Percoll-purified motile spermatozoa and incubated for $20 \mathrm{~h}$ at $38.5{ }^{\circ} \mathrm{C}$ under $5 \% \mathrm{CO}_{2}$ in humidified air. After fertilization, presumptive zygotes ( $n=20$ per treatment; defined below) were vortexed for $6 \mathrm{~min}$ to completely remove the excess sperm and cumulus cells and washed zygotes cultured in potassium simplex optimization medium (KSOM; Specialty Media, Phillipsburg, NJ, USA) supplemented with $0.3 \% \mathrm{BSA}$ at $38.5^{\circ} \mathrm{C}$ in humidified air and $5 \% \mathrm{CO}_{2}$. The embryos were then transferred into fresh KSOM with $0.3 \%$ BSA and $10 \%$ fetal bovine serum at $72 \mathrm{~h}$ after insemination and further cultured until the BL stage (day 7 ).

\section{Sample collection for RNA analysis and immunostaining}

GV- and metaphase II (MII)-stage oocytes were collected immediately after aspiration and $24 \mathrm{~h}$ after in vitro maturation respectively. Both GV- and MII-stage oocytes were stripped of cumulus cells by vortexing for $5 \mathrm{~min}$ in HEPES-TALP containing $0.1 \%$ hyaluronidase. At $\sim 20 \mathrm{~h}$ after fertilization, presumptive zygotes were denuded by vortexing, washed, and used for experimental analysis. In addition, embryos at twocell, four-cell, eight cell, and 16-cell stage were collected at 33, 44,52 , and $72 \mathrm{~h}$ after fertilization respectively, and morula (MO) and BLs were collected at 5 and 7 days after fertilization 
respectively. For each stage of collected oocytes and embryos, four replicates were taken for real-time PCR analysis and immunofluorescent staining.

\section{Quantitative real-time RT-PCR}

Procedures used for RNA isolation, cDNA synthesis, and quantitative real-time RT-PCR to analyze the transcript abundance in GV- and MII-stage oocytes and early embryo samples ( $n=4$ pools of ten oocytes per embryo per group) were carried out as described previously (Bettegowda et al. 2006). RPS18 was used as endogenous control to normalize the gene expression data. See Table 1 for the list of primer sequences used in real-time PCR. For all genes, no template controls showed no amplification and melting curves were run for all samples showing a single product.

\section{Immunofluorescent staining}

Immunofluorescent staining was performed for USF1 protein localization in oocytes and embryos according to protocols published previously (Tripurani et al. 2011). After fixation, permeabilization and blocking, samples were probed with a commercially available rabbit polyclonal anti-USF1 antibody (1:200 (v/v), Santa Cruz Biotechnology, sc229) and the corresponding FITC-conjugated secondary antibody $(0.5 \%$ (w/v) F9887; Sigma-Aldrich). Immunostaining experiments were replicated four times using at least 10-12 oocytes/embryo per sample. Oocytes and embryos incubated without the primary USF1 antibodies were used as negative controls.

\section{Identification of USF1 transcript origin in early embryos}

To determine the origin of USF1 transcripts in early embryos (maternal vs zygotic), presumptive zygotes were cultured in KSOM containing $0.3 \% \mathrm{BSA}$ in the presence and absence of a transcription inhibitor $(50 \mu \mathrm{g} / \mathrm{ml} \boldsymbol{\alpha}$-amanitin). After $52 \mathrm{~h}$ of in vitro culture, pools of eight-cell embryos were collected within treatment $(n=10$ embryos per pool; $n=4$ replicates for $\alpha$-amanitin and untreated controls) and subjected to real-time quantification of USF1 transcript abundance as described earlier.

\section{Design and validation of siRNA-mediated USF1 knockdown}

Microinjection of USF1 siRNA was performed to knockdown endogenous USF1 in bovine embryos using our procedures published previously (Bettegowda et al. 2007). Two distinct siRNA species were designed against the open reading frame of bovine USF1 mRNA using publicly available siRNA design algorithm (siRNA target finder; Ambion, Austin, TX, USA; species 1: sense 5'-AATTCCTCCCTGCAACACTTCCCTGTCTC-3' and antisense 5'-AAGAAGTGTTGCAGGGAGGAACCTGTCTC-3' and species 2: sense 5'-AACATCATTGTCCAGCTGTAGCCTGTCTC- $3^{\prime}$ and antisense 5'-AACTACAGCTGGACAATGATGCCTGTCTC-3'). All candidate siRNA were interrogated using the basic local alignment tool program (http://blast.ncbi.nlm.nih.gov/Blast.cgi) to rule out homology off targets to any other known genes in the bovine expressed sequence tag and genomic database. The USF1 siRNA species were synthesized with the Silencer siRNA construction kit (Ambion) by following the manufacturer's protocol.

Microinjection of USF1 siRNA into bovine embryos and subsequent early embryo culture experiments were conducted as described previously (Bettegowda et al. 2007). Individual USF1 siRNA were validated for specificity and efficacy of USF1 mRNA and protein knockdown in early embryos. Presumptive zygotes collected at 16-18 h after insemination (hpi) were microinjected with $50 \mu \mathrm{M}$ cocktail of USF1 siRNA species (USF1-siRNA-S1 and S2) and four-cell embryos were collected at 42-44 hpi for real-time PCR analysis of USF1 mRNA. Uninjected embryos and embryos injected with negative control siRNA (Universal control species 1; Ambion) were used as control groups $(n=4$ experimental replicates with ten embryos per group). Efficiency of USF1 siRNA species in reducing USF1 protein was determined by USF1 immunostaining of eight-cell embryos collected $72 \mathrm{hpi}(n=10$ embryos for uninjected, negative control siRNA injected, and USF1 siRNA species injected groups).

\section{Effect of USF1 depletion on embryonic development}

To determine the effect of USF1 ablation on early embryonic development, the proportion of USF1 siRNA-injected presumptive zygotes that cleaved $48 \mathrm{hpi}$, reached 8-16C (72 hpi)

Table 1 Primers used for qRT-PCR

\begin{tabular}{|c|c|c|}
\hline Gene name & Accession number & Primer sequence $\left(5^{\prime} \rightarrow 3^{\prime}\right)$ \\
\hline USF1 & NM_001001161 & $\begin{array}{l}\text { F: TGGCCGGACTTAGCACTCA } \\
\text { R: TCCGAGGAACTGGTCCTTCTT }\end{array}$ \\
\hline USF2 & NM_001001162 & $\begin{array}{l}\text { F: AAGGAGAGCTCAGCATAATGAAGTG } \\
\text { R: GAAAGCTGGACGATCCAGTTG }\end{array}$ \\
\hline TWIST2 & NM_001083748 & $\begin{array}{l}\text { F: TCGCCGGTCCATCTCTCA } \\
\text { R: AAGGTTGGGTCTATGAACACTCTCA }\end{array}$ \\
\hline$J Y 1$ & NM_001110098 & $\begin{array}{l}\text { F: TTGGAACTTCCATGGACGACC } \\
\text { R: ATTTGCTGGTGATCCCAAGAG }\end{array}$ \\
\hline GDF9 & NM_174681.2 & $\begin{array}{l}\text { F: GGAATCTGAGGCTGAGACTTGG } \\
\text { R: GTGAAAAGCCTGAGCACTTGTG }\end{array}$ \\
\hline FST & BF774514 F & $\begin{array}{l}\text { F: CAGAGCTGCAAGTCCAGTACCA } \\
\text { R: CATGTAGAGCTGCCTGGACAGA }\end{array}$ \\
\hline RPS18 & NM_001033614 & $\begin{array}{l}\text { F: GTGGTGTTGAGGAAAGCAGACA } \\
\text { R: TGATCACACGTTCCACCTCATC }\end{array}$ \\
\hline
\end{tabular}


and BL stage (7 days after insemination) were recorded and compared with uninjected and negative control siRNA injected embryos. Each experiment was replicated six times with 50 embryos per treatment.

\section{Effect of USF1 depletion on oocyte maturation, cumulus expansion, and abundance of specific oocyte-expressed transcripts}

To determine the functional requirement of USF1 for meiotic maturation, cumulus expansion, and knockdown effects on mRNA abundance for select E-box-containing genes, cumulusenclosed GV-stage oocytes were microinjected with USF-1 siRNA cocktail and pre-incubated for $48 \mathrm{~h}$ in $50 \mu \mathrm{M}$ $S$-roscovitine-containing maturation medium to arrest meiotic maturation and allow sufficient time for USF1 knockdown using our procedures published previously (Lee et al. 2014a). Then, COCs were washed thoroughly in maturation media to completely remove the $S$-roscovitine and subjected to meiotic maturation for $24 \mathrm{~h}$ as described earlier. Uninjected and negative control siRNA-injected COCs were also subjected to the same S-roscovitine treatment and used as control groups. After $24 \mathrm{~h}$ of in vitro maturation, effects of treatments on cumulus expansion and progression to MII were determined ( $n=20$ oocytes/group; $n=4$ replicates) as we described previously (Lee et al. 2014a,b,c). Additional oocytes were stripped of cumulus cells and used for real-time quantification of transcripts for USF1 and related bHLH transcription factors (USF2 and TWIST2) and select genes linked to bovine oocyte competence (JY1, GDF9, and FST; $n=10$ oocytes/ group; $n=4$ replicates).

\section{Statistical analysis}

Relative expression of mRNA was analyzed in SAS using a one-way ANOVA with differences in means detected by Fisher's protected least significant difference (PLSD). Embryo development data were calculated based on the total number of zygotes and was arc-sine transformed before analysis using a one-way ANOVA. A similar statistical analysis as for embryos was used for determination of effects of treatments on meiotic maturation and cumulus expansion. Differences between means were also detected using PLSD. All data are presented untransformed.

\section{Results}

\section{Expression characterization of USF1 mRNA and protein at different stages of oocyte and early embryonic development}

Quantitative real-time PCR was performed to characterize temporal changes in abundance of USF1 transcripts during meiotic maturation and early embryonic development. USF1 mRNA abundance was quite stable during meiotic maturation and pronuclear (PN) stage, increased $(P<0.001)$ at two-cell stage relative to the MII-stage, and then was considerably decreased $(<0.007)$ after the eight-cell stage to barely detectable levels at the BL stage (Fig. 1A). To determine the origin of the USF1 transcripts (maternal vs zygotic) present in early embryos, zygotes were cultured in the presence of the transcriptional inhibitor $\alpha$-amanitin (Fig. 1B) and mRNA abundance was analyzed by real-time PCR at eight-cell stage. The abundance of USF1 transcripts was higher in $\alpha$-amanitin-treated embryos compared with control eight-cell stage embryos, indicating that transcripts detected were maternal in origin and also suggests that embryonic transcription is necessary for normal degradation of USF1 mRNAs after eight-cell stage of embryonic development.

The abundance and localization of USF1 protein was investigated by immunocytochemical analysis (Fig. 2), which demonstrated increased USF1 protein at MII stage relative to GV stage and minimally decreased USF1 protein at zygote and two-cell stage of embryo development. The abundance of USF1 protein increased by the four-cell stage and at eight-cell stage during early embryonic development. After the eight-cell stage, abundance of USF1 protein slightly declined at 16-cell stage and then remained stable during the stages leading up to the BL stage.

\section{USF1 knockdown effect on bovine early embryonic development}

To determine the effect of USF1 depletion on early embryonic development, siRNA-mediated gene silencing procedures were validated in bovine embryos. Microinjection of USF1 siRNA cocktail at the zygote stage of embryo development significantly reduced $(P<0.05)$ the abundance of USF1 mRNA by $\approx 90 \%$ in the resulting four-cell stage embryos relative to the uninjected and negative control siRNA-injected embryos as determined by real-time PCR (Fig. 3A) To further confirm the efficacy of USF1 siRNA, USF1
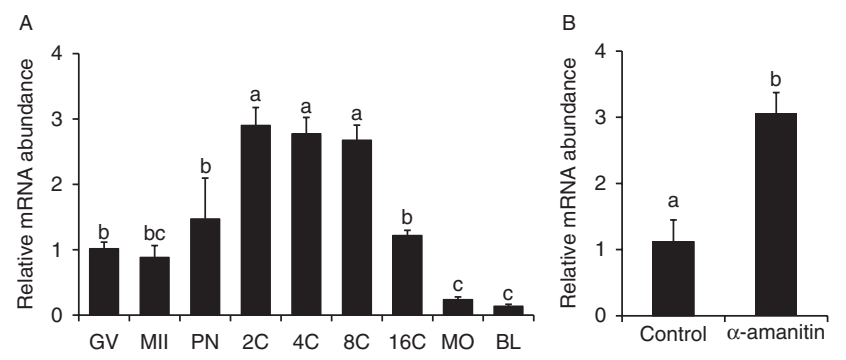

Figure 1 Expression characterization of USF1 mRNA during oocyte and early embryonic development. (A) Relative abundance of USF1 transcript during meiotic maturation (GV and MII stage of oocytes) and at different developmental stages of early embryos (pronuclear (PN), two-cell (2C), four-cell (4C), eight-cell (8C), 16-cell (16C), MO, and blastocyst (BL) stage). (B) Relative amount of USF1 mRNA in eight-cell embryos cultured in the presence of $\alpha$-amanitin is significantly different from that of control embryos. Data were normalized relative to abundance of endogenous control (RPS18) and are expressed as mean \pm S.E.M. Values with different superscripts indicate significant differences $(P<0.05)$. 

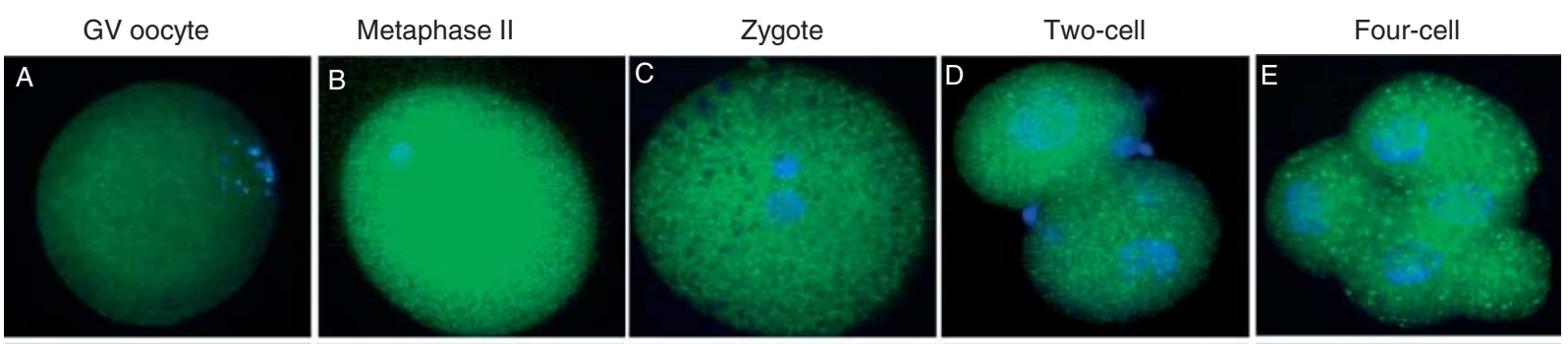

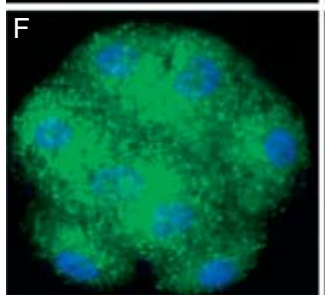

Eight-cell

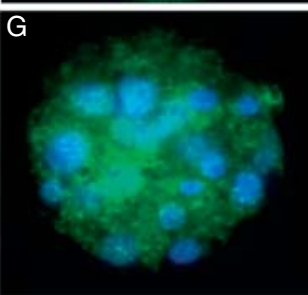

16-cell

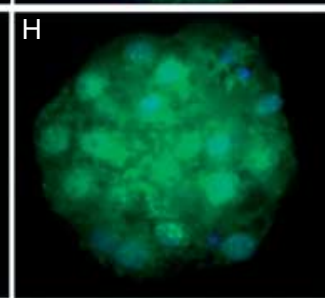

Morula

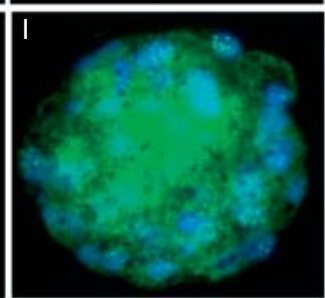

Blastocyst

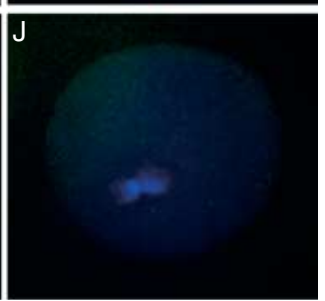

Negative control

Figure 2 Spatiotemporal expression of USF1 protein during oocyte maturation and early embryonic development. Immunofluorescent localization of USF1 protein at different stages of bovine oocyte and preimplantation embryo development. Nuclear DNA was stained with 4',6-diamidino-2phenylindole. Negative control embryos incubated in absence of USF1 antibody.

immunostaining was performed in resulting eight-cell stage embryos. A specific reduction in USF1 protein was observed compared with both uninjected embryos and negative siRNA control-injected embryos (Fig. 3B).

Effect of the USF1 knockdown on early embryonic development was analyzed by microinjection of validated USF1 siRNA cocktail into bovine zygotes and analysis of subsequent effects on cleavage rate, and proportion of embryos developing to eight-cell stage and $\mathrm{BL}$ stages compared with uninjected and negative control siRNA-injected embryos. USF1 siRNA injection did not affect the total cleavage rate (Fig. 4A), but reduced the proportion of IVF embryos developing to the eight-cell stage relative to uninjected and negative control siRNA-injected embryos $(P<0.001$; Fig. 4B). A more than two-fold reduction was observed in the proportion of IVF embryos developing to BL stage for USF1 siRNA-injected embryos compared with uninjected and negative control siRNA-injected embryos $(P<0.001$; Fig. 4C).

\section{Effect of USF1 knockdown in GV stage oocytes on progression to MII, cumulus expansion, and transcript abundance for select oocyte-expressed genes during bovine oocyte maturation in vitro}

Given the observed effect of USF1 siRNA knockdown on early embryonic development, we investigated the functional role of USF1 transcription factor in meiotic maturation, cumulus expansion, and regulation of TWIST2, JY1, GDF9, and FST genes, carrying USF1binding element (E-box) in their promoter region and/or essentially required for the developmental competence of bovine oocytes. Microinjection of USF1 siRNA into GV-stage oocytes did not affect subsequent rates of meiotic maturation and cumulus expansion following in vitro maturation (see Supplementary Table 1, see section on supplementary data given at the end of this article). However, real-time PCR analysis demonstrated that USF1 siRNA injection resulted in a $>95 \%$ decrease in USF1 transcript abundance following $48 \mathrm{~h}$ culture in $S$-roscovitine and the subsequent in vitro maturation $(P<0.0001$; Fig. 5A). TWIST2 and JY1 mRNA abundance were increased in USF1 siRNA-injected oocytes collected at MII stage of meiotic maturation compared with uninjected and negative siRNA control-injected oocytes $(P<0.0003$; Fig. 5B and $C$ respectively). By contrast, GDF9 and FST transcript abundance were reduced in USF1 siRNA-injected oocytes relative to uninjected and negative control siRNA-injected oocytes collected at MII stage $(P<0.0001$; Fig. 5D and $P<0.003$; Fig. 5E respectively). Negative control siRNA injection resulted in a modest decrease in GDF9 transcript abundance compared with uninjected group, suggesting a modest off-target effect of negative control siRNA on GDF9 expression, but GDF9 transcript abundance in USF1 siRNA-injected oocytes was lower than both control groups (uninjected and negative control siRNA injected). These results suggest that USF1 potentially functions as a regulator of GDF9, FST, TWIST2, and JY1 transcript abundance during oocyte maturation. However, abundance of USF2 mRNA was not altered in response to USF1 siRNA injection (Fig. 5F).

\section{Temporal regulation of mRNA for USF2 and TWIST2 transcription factors during oocyte maturation and early embryonic development: Potential link to USF1 action}

Temporal changes in USF2 and TWIST2 transcript abundance were established and compared with results obtained for USF1 to determine potential association of 
A

USF1

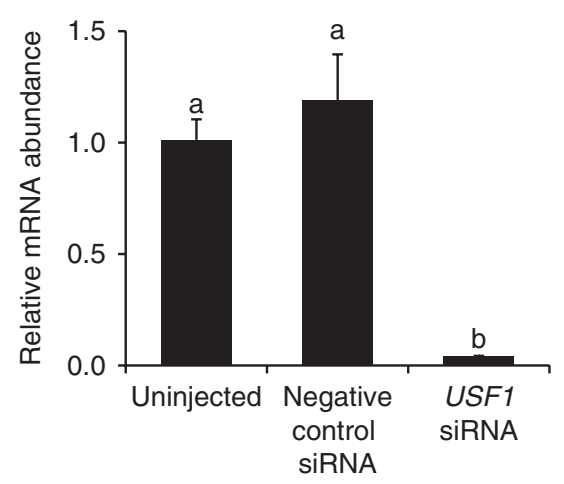

B Uninjected Negative control siRNA
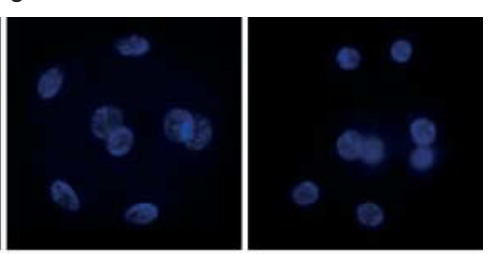

USF1
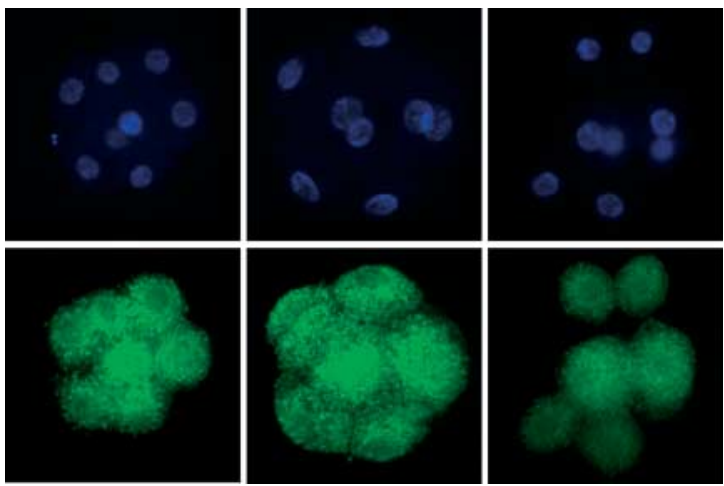

Figure 3 Effect of zygote USF1 siRNA microinjection on USF1 mRNA and protein level in resulting four- and eight-cell embryos respectively. (A) Effect of siRNA microinjection at the pronuclear stage of embryo on abundance of USF1 transcript in resulting four-cell embryos (ten embryos per group, four replicates). Data were normalized relative to abundance of endogenous control RPS18 and are expressed as mean \pm S.E.M. Values with different letters indicate significant difference $(P<0.05)$. (B) Effect of RNAi-mediated depletion of USF1 in bovine zygotes on USF1 protein level in resulting eight-cell embryos as determined by immunofluorescent staining $(n=4)$. Uninjected embryos and embryos injected with a nonspecific siRNA (negative control siRNA) served as controls. Nuclear DNA was stained with 4',6-diamidino-2-phenylindole (DAPI).

these transcription factors (Garcia-Sanz et al. 2013) in transcriptional regulation of $J Y 1, G D F 9$, and other genes containing multiple E-box-binding elements in their promoter regions. In comparison with USF1, USF2 transcript abundance displayed similar changes during oocyte and early embryonic development (Figs $1 \mathrm{~A}$ and $6 \mathrm{~A}$ respectively). On the other hand, temporal changes in TWIST2 mRNA abundance were distinct from those observed for USF1 and USF2 (Fig. 6B). Abundance of TWIST2 mRNA was elevated during meiotic maturation $(P<0.0001)$, decreased $(P<0.0005)$ at the pronuclear and two-cell stages relative to the MII stage, and then significantly increased $(P<0.05)$ by four-cell stage. After four-cell stage, the abundance of TWIST2 transcripts progressively decreased through the $16 \mathrm{C}$ stage and was barely detectable at $\mathrm{MO}$ and $\mathrm{BL}$ stages.

\section{Discussion}

Results of this study established temporal expression of USF1 mRNA and protein during bovine oocyte maturation and early embryogenesis and a functional requirement of this bovine oocyte-expressed USF1 transcription factor for early embryogenesis and regulation of genes involved in oocyte competence and early embryonic development. However, results suggest that USF1 knockdown in GV-stage oocytes does not affect meiotic maturation and cumulus expansion. To our knowledge, a functional role for USF1 in early embryogenesis and potential regulation of genes linked to oocyte competence has not been reported previously.

USF1 is a member of the bHLH leucine zipper family, which has been implicated in tissue-specific transcriptional regulation of a wide range of genes (Littlewood \& Evan 1998). Most commonly, this transcription factor binds to a pyrimidine-rich initiator (Inr) element or E-box motifs of the promoter region as homodimers or a heterodimer (USF1/USF2) and might act with or without association with other E-box-binding transcription factors of bHLH family (Garcia-Sanz et al. 2013). The USF1/USF2 heterodimer is a major DNA-binding form present in most tissues and cell types compared with the homodimers (Fair et al. 1995, Rajput et al. 2013). In our experiments, we observed similar temporal changes in transcript abundance for USF1 and USF2 in bovine oocytes and early embryos, suggesting a potential functional role of USF1/USF2 heterodimer in transcriptional regulation during oocyte and early embryogenesis. However, establishment of USF1/USF2 proteinprotein interactions and a functional role of USF2 in bovine oocytes and early embryos will require further investigation. TWIST2 transcription factor also functions
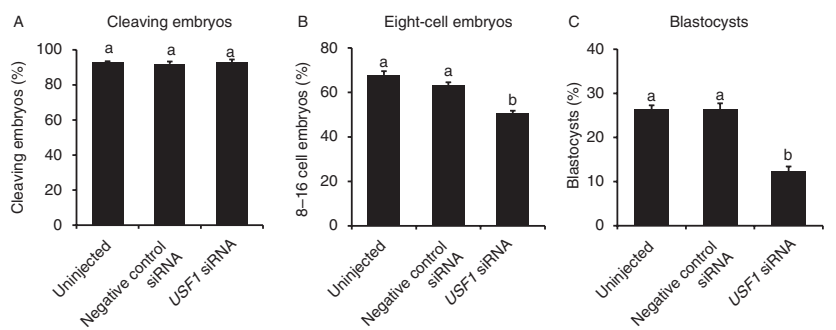

Figure 4 Requirement of USF1 for bovine early embryonic development. Presumptive zygotes were microinjected (16-18 h after fertilization) with either USF1 siRNA (cocktail) or universal negative control siRNA (negative control siRNA) species 1 (Ambion, Austin, TX, USA) or served as uninjected controls. Presumptive zygotes were then cultured in KSOM medium supplemented with $0.3 \%$ BSA (50 presumptive zygotes/group, six replicates). The 8-16-cell stage embryos were separated $72 \mathrm{~h}$ after insemination (hpi) and cultured in fresh KSOM medium supplemented with $0.3 \%$ BSA and $10 \%$ FBS until day 7 . Effect of USF1 depletion on (A) total cleavage rate (determined $48 \mathrm{hpi}$ ), (B) proportion of embryos developing to the $8-16$-cell stage (72 hpi), and (C) proportion of embryos developing to the blastocyst stage (day 7). Data are expressed as mean \pm S.E.M. Values with different superscripts across treatments indicate significant differences $(P<0.001)$. 

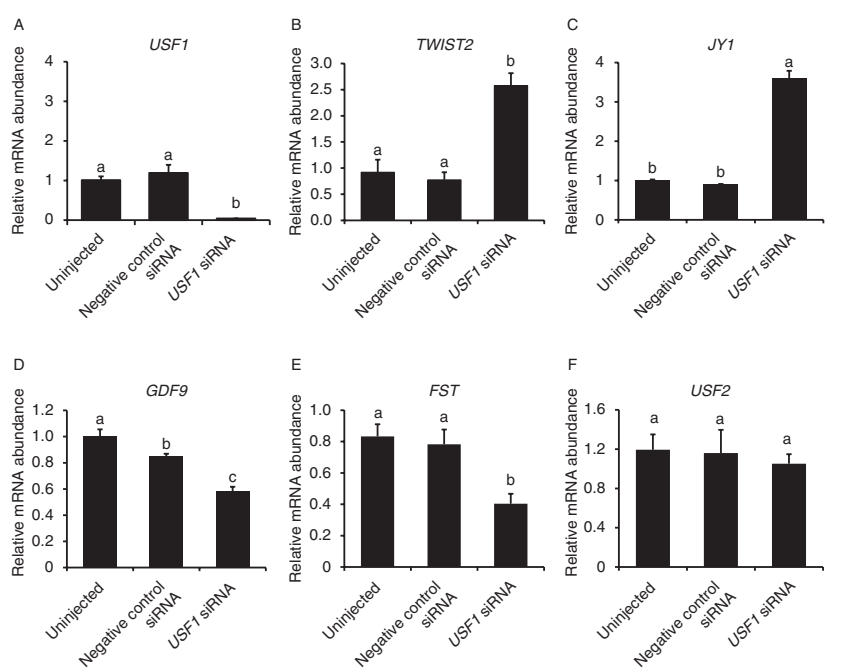

Figure 5 USF1 siRNA injection in GV-stage oocytes affects the mRNA abundance of specific oocyte-expressed genes in resulting MII stage oocytes following in vitro maturation. Samples of cDNA collected from MII-stage oocytes were subjected to real-time RT-PCR analysis for quantification of USF1 (A), TWIST2 (B), JY1 (C), GDF9 (D), FST (E), and USF2 (F) mRNA. Data were normalized relative to abundance of endogenous control RPS18 and are expressed as mean \pm s.E.M. Values with different letters indicate statistical difference $(P<0.0001)$.

as a molecular switch to either activate or repress target genes via direct binding to conserved E-box sequences in the promoter region and recruitment of co-activator(s) or repressor(s) (Franco et al. 2011). High abundance of TWIST2 transcript at MII stage in oocytes and four to eight-cell embryonic stage suggests a potential regulatory role in control of genes associated with maturation and initial cleavage divisions during early embryonic development. Interestingly, many of the mammalian target genes for TWIST2 includes cell adhesion molecules (E-cadherin and fibronectin) and transcription factors ( $\beta$-catenin (CTNNB1) and STAT3; Fang et al. 2011), genes involved in sperm and oocyte fusion (Takezawa et al. 2011), cleavage, compaction, and formation of a implantation competent embryo (Bloor et al. 2002).

The observed significant reduction in both USF1 and USF2 transcripts immediately after embryonic genome activation (occurs at eight-cell stage in bovine) suggests a maternal origin of these transcripts with potential depletion or degradation upon embryonic genome activation. Increased abundance of USF1 transcripts in transcriptional inhibitor ( $\boldsymbol{\alpha}$-amanitin)-treated embryos at eight-cell stage (Fig. 1B) further confirmed their maternal origin and that USF1 transcript depletion is dependent on zygotic degradation machinery activated with the onset of embryonic genome activation. A large number of maternal transcripts are degraded by zygotic decay machinery during early embryonic development in Drosophila and other bilateria including zebrafish, frogs, and mice (Tadros \& Lipshitz 2009, Thomsen et al. 2010). We have observed a similar transcription-dependent degradation process of maternal transcripts for TGF $\beta$ superfamily signaling pathway components in bovine embryos coincident with genome activation (Lee et al. 2014b). Results suggest that timely depletion of maternal transcription factors and signaling proteins is associated with the progression of embryo development.

We investigated the functional role of USF1 during meiotic maturation and early embryonic development using an effective and previously optimized siRNAbased gene knockdown approach for oocytes and embryos (Bettegowda et al. 2007, Lee et al. 2014c). siRNA-based knockdown of USF1 transcripts in fertilized embryos resulted in effective reduction in proportion of embryos reaching the eight-cell stage and the BL stage. However, no effect was observed on total cleavage rates, which suggest that USF1 transcription factor is potentially required for later stages of embryogenesis after initial cleavage division. Results suggest that USF1 is potentially required for development events at or after the eight-cell stage including embryonic genome activation. Moreover, USF1 contains a nuclear localization signal that directs its transport to the nucleus to regulate a broad spectrum of targeted gene transcription (McMurray \& McCance 2003, Crusselle-Davis et al. 2006, Anantharaman et al. 2011). The transcriptional regulation through USF1 has previously been shown to be associated with its nuclear abundance in various cellular models (Zhang et al. 2007, van Deursen et al. 2008, Viswanathan et al. 2009). Therefore, it is likely that increased abundance of USF1 protein in eight-cell stage embryos (Fig. 2) coincident with embryonic genome activation is of potential functional importance in the transcriptional activity of the embryonic genome.

Results of this study provide support for a functional role of USF1 transcription factors in regulation of multiple genes linked to oocyte competence in cattle. In bovine, JY1 and GDF9 are widely studied oocytespecific genes, which contain multiple E-boxes in their regulatory region and play an important role in oocyte
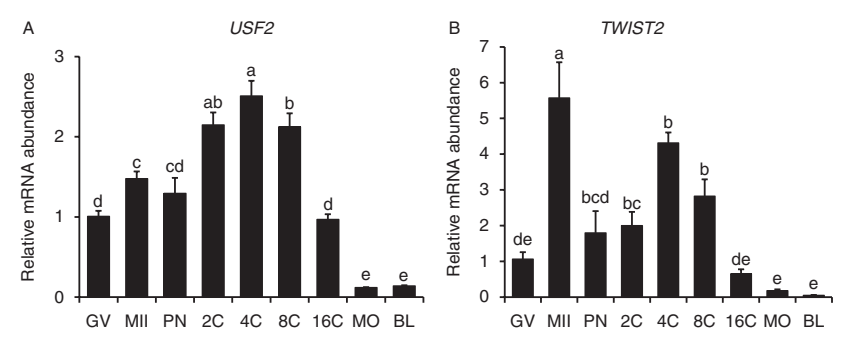

Figure 6 Expression characterization of USF2 and TWIST2 mRNA during oocyte and early embryonic development. Real-time PCR analysis for quantification of USF2 (A) and TWIST2 (B) mRNA during meiotic maturation (GV and MII stage of oocytes) and at different developmental stages of early embryos (pronuclear (PN), two-cell (2C), four-cell (4C), eight-cell (8C), 16-cell (16C), MO, and blastocyst (BL) stage). Transcript levels were normalized relative to abundance of endogenous control (RPS18) RNA and are expressed as mean \pm S.E.M. Time points without a common letter (a, b, c, d, and e) are significantly different $(P<0.05)$. 
competence for successful early embryonic development (Yan et al. 2006, Bettegowda et al. 2007, Roy et al. 2013, Lee et al. 2014a). Our results demonstrated that siRNA-mediated depletion of USF1 in GV-stage oocytes significantly reduced the transcript abundance for GDF9 gene at MII stage, which suggests a positive role in regulation of GDF9 mRNA abundance and presumably transcription. NOBOX and E-box motifs are the critical regulatory sequences, found in the GDF9 promoter, responsible for its oocyte specific expression in mammals (Rajkovic et al. 2004, Yan et al. 2006). Functional analysis of promoter mutations has established an essential E-box regulatory sequence (CAGCTG) at -182 to -177 bp upstream of the Gdf9 initiation codon in mice, which was found to be conserved across the 16 mammalian species including cattle (Bettegowda et al. 2007). Interestingly, it has been demonstrated that USF1 transcription factor can bind to E-box motif (CAGCTG) to regulate tissue-specific transcription in mice (Fang et al. 2011).

Results support potential USF1-dependent regulation of GDF9 transcription in bovine oocytes, but further studies are needed to conclusively prove transcriptional regulation. In cattle, oocytes in the growth phase display the greatest transcriptional activity (Fair et al. 1995), but direct evidence of transcriptional activity during meiotic maturation is limited. Array analysis of the bovine oocyte transcriptome revealed increased abundance of $\sim 10 \%$ of the mRNAs during meiotic maturation (Dalbies-Tran \& Mermillod 2003). This increase in mRNA abundance was potentially attributed to transcriptional activity at the beginning of meiotic maturation of GV oocytes aspirated from $>3 \mathrm{~mm}$ antral follicles and cultured in vitro (Memili et al. 1998, Hendriksen et al. 2000, Tomek et al. 2002). Moreover, $24 \mathrm{~h}$ incubation in the presence of $C D K$ inhibitors to maintain meiotic arrest resulted in increased content of poly $(\mathrm{A}) \mathrm{mRNAs}$ in bovine oocyte (Lequarre et al. 2004). In these studies, we incubated USF1 siRNA-injected oocytes in the presence of the CDK inhibitor roscovitine for $48 \mathrm{~h}$ to allow time for siRNAmediated depletion of USF1 before meiotic maturation. Therefore, it is possible that USF1 transcription factor knockdown reduced GDF9 expression during meiotic maturation in vitro through interaction with E-box regulatory elements present in the GDF9 promoter. However, further studies are required to conclusively establish USF1-mediated transcriptional regulation of the GDF9 gene in bovine oocytes.

Results from our previous studies have demonstrated an important functional requirement of the bovine oocyte-specific JY1 gene for meiotic maturation and early embryogenesis (Bettegowda et al. 2007). However, no effect of USF1 knockdown on meiotic maturation and cumulus expansion was observed in the current studies. Depletion of $J Y 1$ transcripts in zygote-stage embryos results in considerably reduced rates of development to the 8-16-cell stage and BL compared with controls.
Interestingly, five putative E-box motifs have been identified within $500 \mathrm{bp}$ upstream of the JY1 TSS (Bettegowda et al. 2007). Out of the five putative E-box motifs, three have CAGCTG E-box motif and the one E-box adjoining TSS has a CACGTG motif, a well-known USF transcription factor-binding sequence. In this study, siRNA-based knockdown of USF1 in GV-stage oocytes resulted in a significant increase in $J Y 1$ transcripts in MII-stage oocytes, which suggests a potential negative regulatory role for USF1 in modulation of JY1 transcription. Similar to other bHLH proteins, USF1 function in both transcriptional activation and repression has been previously characterized and activity (activation vs repression) is dependent upon specific binding partner in various cellular models (McMurray \& McCance 2003, Crusselle-Davis et al. 2006, Anantharaman et al. 2011). The presence of multiple E-boxes at $5^{\prime}$ flanking region of the $J Y 1$ promoter suggests the binding of more than one bHLH transcription factor (BHLHE41, TWIST, and USF2) which might be working in association with USF1 to repress $/ Y 1$ transcription in oocytes.

Additionally, we have also analyzed the effect of USF1 knockdown on abundance of other transcripts linked to bovine oocyte and embryo competence (FST) and potential USF1-binding partners (USF2 and TWIST2) in USF1 siRNA-injected oocyte collected at MII stage. FST has been previously characterized as an important maternal (oocyte-derived) factor required for early embryo cleavage, BL development, and functional determinant of BL cell allocation (Rajput et al. 2013). Reduced expression of FST in USF1-depleted oocytes potentially supports the observed functional role of USF1 TF in early embryonic development via regulating the transcription of oocyte-expressed genes. Furthermore, siRNA-mediated depletion of USF1 enhanced the mRNA abundance for TWIST2, whereas abundance of USF2 mRNA remained unchanged in MII-stage oocytes. In support of USF1 knockdown effects reported, in silico analysis revealed five different E-boxes within $1.3 \mathrm{~kb}$ upstream of the TWIST2 mRNA TSS and two of them (CACCTG and CAGATG) were found to be highly conserved among mammals (unpublished). Moreover, two putative E-boxes were identified within the $5^{\prime}$-UTR region of the FST gene and one (CACCTG) showed high level of conservation, whereas E-box motifs were not detected upstream of the USF2 TSS (Sandeep K Rajput and George W Smith, unpublished observations). Collectively, these results suggest that USF1 TF potentially mediates E-box-dependent positive regulation of GDF9 and FST, and negative regulation of TWIST2 and JY1 transcription.

In conclusion, results of this study provide novel information about the spatiotemporal expression pattern of USF1, USF2, and TWIST transcription factors during oocyte and early embryonic development. Results establish a functional requirement of USF1 transcription factor in embryo development to the BL stage and 
suggest an important regulatory role of USF1 transcription factor in modulating the transcription of GDF9, FST, TWIST2, and JY1 genes in bovine oocytes. Moreover, siRNA-mediated depletion of USF1 transcription factor resulted in a similar adverse effect on early embryonic development as observed upon JY1 and FST knockdown in fertilized embryos. Further mutation analysis of the GDF9, FST, TWIST2, and JY1 promoter containing regulatory E-boxes and their interaction with USF1 and other associated bHLH transcription factors will provide further insight into precise molecular mechanisms involved in oocyte/embryo transcriptional regulation in cattle.

\section{Supplementary data}

This is linked to the online version of the paper at http://dx.doi. org/10.1530/REP-14-0445.

\section{Declaration of interest}

The authors declare that there is no conflict of interest that could be perceived as prejudicing the impartiality of the research reported.

\section{Funding}

This study was supported by the National Research Initiative competitive grant number 2008-35203-19094 from the U.S.D.A. National Institute of Food and Agriculture and the National Institute of Child Health and Human Development of the National Institutes of Health under award number R01HD072972. T K Datta was supported by the travel grant from the Department of Biotechnology, Government of India.

\section{References}

Anantharaman A, Lin IJ, Barrow J, Liang SY, Masannat J, Strouboulis J, Huang S \& Bungert J 2011 Role of helix-loop-helix proteins during differentiation of erythroid cells. Molecular and Cellular Biology 31 1332-1343. (doi:10.1128/MCB.01186-10)

Bettegowda A, Patel OV, Ireland JJ \& Smith GW 2006 Quantitative analysis of messenger RNA abundance for ribosomal protein L-15, cyclophilin-A, phosphoglycerokinase, $\beta$-glucuronidase, glyceraldehyde 3-phosphate dehydrogenase, $\beta$-actin, and histone $\mathrm{H} 2 \mathrm{~A}$ during bovine oocyte maturation and early embryogenesis in vitro. Molecular Reproduction and Development 73 267-278. (doi:10.1002/mrd.20333)

Bettegowda A, Yao J, Sen A, Li Q, Lee KB, Kobayashi Y, Patel OV, Coussens PM, Ireland JJ \& Smith GW 2007 JY-1, an oocyte-specific gene, regulates granulosa cell function and early embryonic development in cattle. PNAS 104 17602-17607. (doi:10.1073/pnas.0706383104)

Bloor DJ, Metcalfe AD, Rutherford A, Brison DR \& Kimber SJ 2002 Expression of cell adhesion molecules during human preimplantation embryo development. Molecular Human Reproduction 8 237-245. (doi:10.1093/molehr/8.3.237)

Bultman SJ, Gebuhr TC, Pan H, Svoboda P, Schultz RM \& Magnuson T 2006 Maternal BRG1 regulates zygotic genome activation in the mouse. Genes \& Development 20 1744-1754. (doi:10.1101/gad.1435106)

Burns KH, Viveiros MM, Ren Y, Wang P, DeMayo FJ, Frail DE, Eppig JJ \& Matzuk MM 2003 Roles of NPM2 in chromatin and nucleolar organization in oocytes and embryos. Science 300 633-636. (doi:10. 1126/science.1081813)
Corre S \& Galibert MD 2005 Upstream stimulating factors: highly versatile stress-responsive transcription factors. Pigment Cell Research 18 337-348. (doi:10.1111/j.1600-0749.2005.00262.x)

Crusselle-Davis VJ, Vieira KF, Zhou Z, Anantharaman A \& Bungert J 2006 Antagonistic regulation of $\beta$-globin gene expression by helix-loop-helix proteins USF and TFII-I. Molecular and Cellular Biology 26 6832-6843. (doi:10.1128/MCB.01770-05)

Dalbies-Tran R \& Mermillod P 2003 Use of heterologous complementary DNA array screening to analyze bovine oocyte transcriptome and its evolution during in vitro maturation. Biology of Reproduction 68 252-261. (doi:10.1095/biolreprod.102.007872)

Dean J 1992 Biology of mammalian fertilization: role of the zona pellucida. Journal of Clinical Investigation 89 1055-1059. (doi:10.1172/ JCI115684)

De Sousa PA, Caveney A, Westhusin ME \& Watson AJ 1998 Temporal patterns of embryonic gene expression and their dependence on oogenetic factors. Theriogenology 49 115-128. (doi:10.1016/S0093691X(97)00406-8)

Dong J, Albertini DF, Nishimori K, Kumar TR, Lu N \& Matzuk MM 1996 Growth differentiation factor-9 is required during early ovarian folliculogenesis. Nature 383 531-535. (doi:10.1038/383531a0)

van Deursen D, Jansen H \& Verhoeven AJ 2008 Glucose increases hepatic lipase expression in HepG2 liver cells through upregulation of upstream stimulatory factors 1 and 2. Diabetologia 51 2078-2087. (doi:10.1007/ s00125-008-1125-6)

Fair T, Hyttel P \& Greve T 1995 Bovine oocyte diameter in relation to maturational competence and transcriptional activity. Molecular Reproduction and Development 42 437-442. (doi:10.1002/mrd.1080420410)

Fang X, Cai Y, Liu J, Wang Z, Wu Q, Zhang Z, Yang CJ, Yuan L \& Ouyang G 2011 Twist2 contributes to breast cancer progression by promoting an epithelial-mesenchymal transition and cancer stem-like cell selfrenewal. Oncogene 30 4707-4720. (doi:10.1038/onc.2011.181)

Franco HL, Casasnovas J, Rodriguez-Medina JR \& Cadilla CL 2011 Redundant or separate entities? - roles of Twist1 and Twist2 as molecular switches during gene transcription Nucleic Acids Research 39 1177-1186. (doi:10.1093/nar/gkq890)

Garcia-Sanz P, Fernandez-Perez A \& Vallejo M 2013 Differential configurations involving binding of USF transcription factors and Twist1 regulate Alx3 promoter activity in mesenchymal and pancreatic cells. Biochemical Journal 450 199-208. (doi:10.1042/BJ20120962)

Hendriksen PJ, Vos PL, Steenweg WN, Bevers MM \& Dieleman SJ 2000 Bovine follicular development and its effect on the in vitro competence of oocytes. Theriogenology 53 11-20. (doi:10.1016/S0093-691X (99)00236-8)

Hutt KJ, McLaughlin EA \& Holland MK 2006 Kit ligand and c-Kit have diverse roles during mammalian oogenesis and folliculogenesis. Molecular Human Reproduction 12 61-69. (doi:10.1093/molehr/ gal010)

Joshi S, Davies H, Sims LP, Levy SE \& Dean J 2007 Ovarian gene expression in the absence of FIGLA, an oocyte-specific transcription factor. BMC Developmental Biology 7 67. (doi:10.1186/1471-213X-7-67)

Lee KB, Bettegowda A, Wee G, Ireland JJ \& Smith GW 2009 Molecular determinants of oocyte competence: potential functional role for maternal (oocyte-derived) follistatin in promoting bovine early embryogenesis. Endocrinology 150 2463-2471. (doi:10.1210/en.2008-1574)

Lee KB, Wee G, Zhang K, Folger JK, Knott JG \& Smith GW 2014 a Functional role of the bovine oocyte-specific protein JY-1 in meiotic maturation, cumulus expansion, and subsequent embryonic development. Biology of Reproduction 90 69. (doi:10.1095/biolreprod.113. 115071)

Lee KB, Folger JK, Rajput SK \& Smith GW 2014b Temporal regulation of mRNAs for select bone morphogenetic proteins (BMP), BMP receptors and their associated SMAD proteins during bovine early embryonic development: effects of exogenous BMP2 on embryo developmental progression. Reproductive Biology and Endocrinology 12 67. (doi:10. 1186/1477-7827-12-67)

Lee KB, Zhang K, Folger JK, Knott JG \& Smith GW 2014c Evidence supporting a functional requirement of SMAD4 for bovine preimplantation embryonic development: a potential link to embryotropic actions of follistatin. Biology of Reproduction 91 62. (doi:10.1095/biolreprod.114. 120105) 
Lequarre AS, Traverso JM, Marchandise J \& Donnay I 2004 Poly(A) RNA is reduced by half during bovine oocyte maturation but increases when meiotic arrest is maintained with CDK inhibitors. Biology of Reproduction 71 425-431. (doi:10.1095/biolreprod.103.026724)

Liang L, Soyal SM \& Dean J 1997 FIG $\alpha$, a germ cell specific transcription factor involved in the coordinate expression of the zona pellucida genes. Development 124 4939-4947.

Littlewood TD \& Evan GI 1998 Helix-Loop-Helix Transcription Factors, pp. 164. Oxford: Oxford University Press.

McMurray HR \& McCance DJ 2003 Human papillomavirus type 16 E6 activates TERT gene transcription through induction of c-Myc and release of USF-mediated repression. Journal of Virology 77 9852-9861. (doi:10. 1128/JVI.77.18.9852-9861.2003)

Memili E, Dominko T \& First NL 1998 Onset of transcription in bovine oocytes and preimplantation embryos. Molecular Reproduction and Development 51 36-41. (doi:10.1002/(SICl)1098-2795(199809)51:1< 36::AID-MRD4 > 3.0.CO;2-X)

Mottershead DG, Ritter LJ \& Gilchrist RB 2012 Signalling pathways mediating specific synergistic interactions between GDF9 and BMP15. Molecular Human Reproduction 18 121-128. (doi:10.1093/molehr/ gar056)

Mottershead DG, Harrison CA, Mueller TD, Stanton PG, Gilchrist RB \& McNatty KP 2013 Growth differentiation factor 9:bone morphogenetic protein 15 (GDF9:BMP15) synergism and protein heterodimerization. PNAS 110 E2257. (doi:10.1073/pnas.1303459110)

Peng H, Chang B, Lu C, Su J, Wu Y, Lv P, Wang Y, Liu J, Zhang B, Quan F et al. 2012 Nlrp2, a maternal effect gene required for early embryonic development in the mouse. PLOS ONE 7 e30344. (doi:10.1371/journal. pone.0030344)

Peng J, Li Q, Wigglesworth K, Rangarajan A, Kattamuri C, Peterson RT, Eppig JJ, Thompson TB \& Matzuk MM 2013 Growth differentiation factor 9:bone morphogenetic protein 15 heterodimers are potent regulators of ovarian functions. PNAS 110 E776-E785. (doi:10.1073/ pnas.1218020110)

Rajkovic A, Pangas SA, Ballow D, Suzumori N \& Matzuk MM 2004 NOBOX deficiency disrupts early folliculogenesis and oocyte-specific gene expression. Science 305 1157-1159. (doi:10.1126/science. 1099755)

Rajput SK, Lee K, Zhenhua G, Di L, Folger JK \& Smith GW 2013 Embryotropic actions of follistatin: paracrine and autocrine mediators of oocyte competence and embryo developmental progression. Reproduction, Fertility and Development 26 37-47. (doi:10.1071/RD13282)

Roy B, Rajput S, Raghav S, Kumar P, Verma A, Jain A, Jain T, Singh D, De S, Goswami SL et al. 2013 Characterization of oocyte-expressed GDF9 gene in buffalo and mapping of its TSS and putative regulatory elements. Zygote 21 115-124. (doi:10.1017/S0967199411000712)

Saeki H, Ohsumi K, Aihara H, Ito T, Hirose S, Ura K \& Kaneda Y 2005 Linker histone variants control chromatin dynamics during early embryogenesis. PNAS 102 5697-5702. (doi:10.1073/pnas.0409824102)

Soyal SM, Amleh A \& Dean J 2000 FIG $\alpha$, a germ cell-specific transcription factor required for ovarian follicle formation. Development 127 4645-4654.

Sylvestre EL, Robert C, Pennetier S, Labrecque R, Gilbert I, Dufort I, Leveille MC \& Sirard MA 2013 Evolutionary conservation of the oocyte transcriptome among vertebrates and its implications for understanding human reproductive function. Molecular Human Reproduction 19 369-379. (doi:10.1093/molehr/gat006)

Tadros W \& Lipshitz HD 2009 The maternal-to-zygotic transition: a play in two acts. Development 136 3033-3042. (doi:10.1242/dev.033183)
Takezawa Y, Yoshida K, Miyado K, Sato M, Nakamura A, Kawano N, Sakakibara K, Kondo T, Harada Y, Ohnami N et al. $2011 \beta$-catenin is a molecular switch that regulates transition of cell-cell adhesion to fusion. Scientific Reports 1 68. (doi:10.1038/srep00068)

Tanaka M, Hennebold JD, Macfarlane J \& Adashi EY 2001 A mammalian oocyte-specific linker histone gene H1oo: homology with the genes for the oocyte-specific cleavage stage histone ( $\mathrm{cs}-\mathrm{H} 1)$ of sea urchin and the B4/H1M histone of the frog. Development 128 655-664.

Thomsen S, Anders S, Janga SC, Huber W \& Alonso CR 2010 Genome-wide analysis of mRNA decay patterns during early Drosophila development. Genome Biology 11 R93. (doi:10.1186/gb-2010-11-9-r93)

Tomek W, Torner H \& Kanitz W 2002 Comparative analysis of protein synthesis, transcription and cytoplasmic polyadenylation of mRNA during maturation of bovine oocytes in vitro. Reproduction in Domestic Animals 37 86-91. (doi:10.1046/j.1439-0531.2002.00336.x)

Tong ZB, Gold L, Pfeifer KE, Dorward H, Lee E, Bondy CA, Dean J \& Nelson LM 2000 Mater, a maternal effect gene required for early embryonic development in mice. Nature Genetics 26 267-268. (doi:10. 1038/81547)

Tripurani SK, Lee KB, Wang L, Wee G, Smith GW, Lee YS, Latham KE \& Yao J 2011 A novel functional role for the oocyte-specific transcription factor newborn ovary homeobox (NOBOX) during early embryonic development in cattle. Endocrinology 152 1013-1023. (doi:10.1210/en. 2010-1134)

Tsunemoto K, Anzai M, Matsuoka T, Tokoro M, Shin SW, Amano T, Mitani T, Kato H, Hosoi Y, Saeki K et al. 2008 Cis-acting elements (E-box and $\mathrm{NBE}$ ) in the promoter region of three maternal genes (histone $\mathrm{H} 1 \mathrm{oo}$, nucleoplasmin 2, and zygote arrest 1) are required for oocytespecific gene expression in the mouse. Molecular Reproduction and Development 75 1104-1108. (doi:10.1002/mrd.20863)

Viswanathan P, Wood MA \& Walker WH 2009 Follicle-stimulating hormone $(\mathrm{FSH})$ transiently blocks $\mathrm{FSH}$ receptor transcription by increasing inhibitor of deoxyribonucleic acid binding/differentiation-2 and decreasing upstream stimulatory factor expression in rat Sertoli cells. Endocrinology 150 3783-3791. (doi:10.1210/en.2008-1261)

Wu X, Viveiros MM, Eppig JJ, Bai Y, Fitzpatrick SL \& Matzuk MM 2003 Zygote arrest 1 (Zar1) is a novel maternal-effect gene critical for the oocyte-to-embryo transition. Nature Genetics 33 187-191. (doi:10. 1038/ng1079)

Xue G \& Hemmings BA 2012 Phosphorylation of basic helix-loop-helix transcription factor twist in development and disease. Biochemical Society Transactions 40 90-93. (doi:10.1042/BST20110678)

Yan C, Elvin JA, Lin YN, Hadsell LA, Wang J, DeMayo FJ \& Matzuk MM 2006 Regulation of growth differentiation factor 9 expression in oocytes in vivo: a key role of the E-box. Biology of Reproduction 74 999-1006. (doi:10.1095/biolreprod.105.050013)

Zhang L, Handel MV, Schartner JM, Hagar A, Allen G, Curet M \& Badie B 2007 Regulation of IL-10 expression by upstream stimulating factor (USF-1) in glioma-associated microglia. Journal of Neuroimmunology 184 188-197. (doi:10.1016/j.jneuroim.2006.12.006)

Received 27 August 2014

First decision 30 September 2014

Revised manuscript received 22 October 2014

Accepted 10 November 2014 